\title{
Phonological rules for a semantics-to-speech system of Japanese: a rule application experiment
}

\author{
Shigeru Sato \\ Informatics Laboratory, Tohoku Institute of Technology, \\ 35-1, Yagiyama-kasumicho, Taihaku, Sendai, 982 Japan
}

(Received 21 June 1989)

\begin{abstract}
A phonetic form generation experiment is performed for the purpose of examining validity of the phonological rules and their application algorithms. The rules reside in the phonological component of a semantics-to-speech system implemented in a computer, and are tiered firstly based on the cyclic rule application principle, and secondly on the two different dimensions of phonological processing: segmental and accentual. Successful integration of application of the segmental and accentual rules is achieved through conversion of tree-structured sentences to phonetic form. The rules are installed in the system in a human-editable format, translated by a rule compiler into executable Lisp functions, tested and found to be valid.
\end{abstract}

PACS number: 43. 72. Ja

\section{INTRODUCTION}

There has long been a constant need for making speech an efficient tool for man-machine communication, with an ultimate goal of the efforts being a machine that acquires human intelligence for handling speech. Construction of a speech synthesis system that includes generation from a semantic base gains importance in this respect. For that reason accurate analysis of relevant phenomena and their description consistent with human linguistic processing are indispensable. Linguistic rules incorporated, speech synthesis systems now current are of two types: (1) speech synthesis from written text (textto-speech synthesis), ${ }^{1,2)}$ and (2) speech synthesis from concept (semantics-to-speech). ${ }^{3-5}$ Although the former has been far better studied and technologically in greater demand, the significance of the latter cannot be neglected for future inclusion of flexibility of human speech.

This paper deals with computer implementation of phonological rules in a semantics-to-speech system and verification of these rules through phonetic form generation experiment. A number of computer im- plementations of semantics-to-speech systems have been reported. The one for English proposed by Young and Fallside ${ }^{3)}$ uses Chomskyan grammar ${ }^{6)}$ to generate sentences, and has an independent phonological component. However, since the output from the syntax is in English orthography, its phonology cannot radically differ from those in textto-speech systems. But ideally, it should result in a different form since in that case, the phonology must include an interfacing mechanism with which to handle input sentences being sequences of treestructured morphemes. A semantics-to-speech system of Japanese by Yamamoto et al. ${ }^{4)}$ does not seem to guarantee mutual independence of the syntax and phonology modules, whose importance should be emphasized not only for human-like operated speech, but also for systematic implementation and management of linguistic knowledge in machines. In our efforts in the semantics-tospeech system of Japanese ${ }^{5,7)}$ emphasis has been placed on: (1) linguistic refinement of the roles carried by the constituent components, and (2) strict modularization of these components to realize transparent rule management required for technological 
feasibility of the system construction. The actual system consists of the following three major parts: (1) the syntactic component (syntax) that generates a sentence structure from a set of conceptual fragments, (2) the phonological component (phonology) that creates a sequence of discrete sounds (phonetic form) from a syntactic tree, and (3) the speech synthesizer that converts the phonetic form into synthesis parameters for speech wave calculation, and produces physical speech.

Novelties of the phonological component we will propose in this paper lies in the following: (1) syntactic structure to phonetic form conversion; it accepts tree structures and produces linearly ordered morpheme sequences, which are phonologically phonetic form, (2) tiered rule ordering based on the cyclic application principle, (3) integration of application of the segmental and accentual rules, and (4) simultaneous execution of structure conversion and rule application. The purpose of this paper is to perform a phonetic form generation experiment to verify validity of the phonological rules ${ }^{7-9)}$ and rule application algorithms employed in this phonological component. The rules are tiered according firstly to the two different dimensions of phonological processing: segmental and accentual, and secondly to their rule-applicational relevance to syntactic structures: cyclic and noncyclic. The algorithms of these applications we will discuss in detail in Sec. 3. To the cyclic segmental rules we grant the role of mora generation and preservation, ${ }^{8)}$ whereas the noncyclic segmental rules deal with phonetic smoothing of segmental sequences. The cyclic accentual rules are meant to realize phrasal accent assignment, whose output the noncyclic accentual rules handle on a syllabic basis toward accent movement among/ within the syllable(s). The rules are ordered cyclic to noncyclic. In the cyclic stage, syntactic trees are convered to linearly aligned phonetic form as the morphological, ${ }^{8)}$ segmental ${ }^{8)}$ and accentual ${ }^{9)}$ rules are applied, whereas noncyclically, the segmental, ${ }^{8)}$ accentual $^{9)}$ and phonetic $^{8)}$ rules scan sequences of phonetic form from left to right.

For computer implementation of the syntactic and phonological components we use Franz-Lisp ${ }^{10)}$ for the reasons that follow: the basic data structure it handles can easily and fully express tree-structured sentences, and hence the data and rules are expected to be programmed in easily readable and maintainable format. The rules are all represented in a con- text-sensitive rewrite rule format, and then forwarded to the rule compiler we have developed to translate them into executable Lisp functions. The results show that the cyclic application algorithm is well capable of handling resolution of tree structures into sequences of phonetic symbols, and that the rules produce appropriate phonetic form from corresponding syntactic structures. Simultaneously executed, cyclic rule application, ${ }^{8,9)}$ together with recursive concatenation of a suffix to a stem, brings about a successful integration of segmental and accentual aspects of phonological processing.

\section{THE SEMANTICS-TO-SPEECH SYSTEM}

For the purpose of confirming validity of the segmental and accentual rules ${ }^{8,9)}$ and examining their integrated operation we will perform a phonetic form experiment in Sec. 3. on a semantics-to-speech system. ${ }^{5,7)}$ This section describes a global structure of the entire system, of which the phonological component is the focus with its tiered rules.

Figure 1 shows a flow diagram of the semanticsto-speech system consisting of three major subsystems indicated by upper case bold face; the syntactic component (syntax) 〈SYNTACTIC GENERATOR> accepts semantic frames which is a structured set of conceptual fragments and yielding a treestructured sentence, the phonological rule component (phonology) 〈PHONOLOGICAL PROCESSOR〉 produces phonetic form corresponding to the input sentence, and the 〈SPEECH SYNTHESIZER〉 renders physical speech. Implementation of the first two of this three-part system has been developed in Franz-Lisp in the task domain of a fairy tale. ${ }^{11)} \mathrm{We}$ chose Franz-Lisp ${ }^{10)}$ firstly because its basic data structure directly and fully corresponds to sentence structure the syntax and phonology deal with, and secondly because programmed rules and computed results are expected to be easily readable and maintainable. Note that the phonology has three tiers of rules in the loop and another three tiers below it, which we call the cyclic and noncyclic rule classes, respectively.

Figure 2 is an example of semantic frame representation $^{12)}$ obtained originally through syntactic, semantic and discourse analysis of a written Esperanto text. ${ }^{5}{ }^{13)}$ The labels 〈unit〉, 〈event〉 and $\langle$ frame $\rangle$ roughly correspond syntactically to a sentence, a clause, and a lexical morpheme, respectively. 


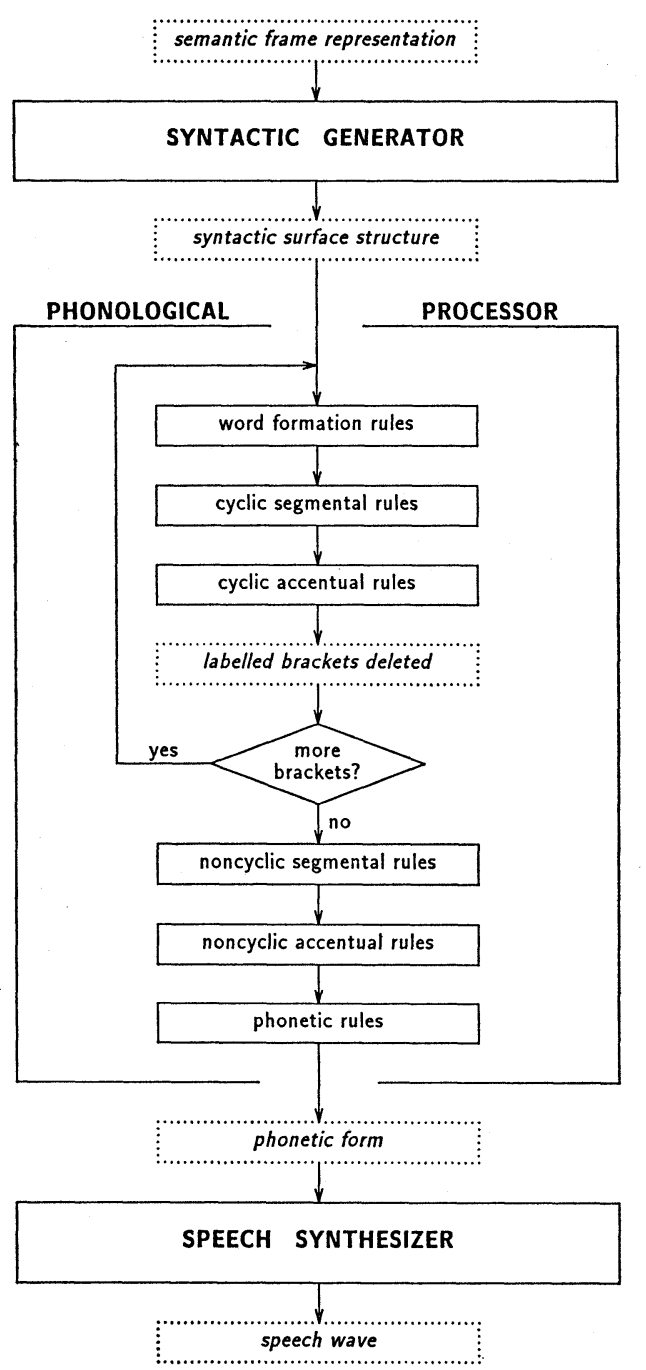

Fig. 1 Flow diagram of the semantics-tospeech system.

A 〈unit> is a chunk cut out of a semantic scene and can contain single or multiple events, whereas an 〈event $\rangle$ is a unit of action and normally consists of multiple $\langle$ frame $\rangle$ 's. Figure 2 is a semantic representation, approximately meaning 'Yuuzi was flying an aeroplane in the field.' In Fig. $2\langle$ unit11〉 contains an event named 〈event1〉, which in turn contains five frames $\langle$ frame1-5 $\rangle$. The frames are from top to bottom: 〈frame2〉 for description of modality of the proposition, $\langle$ frame3 $\rangle$ for lexical verb \{tobas

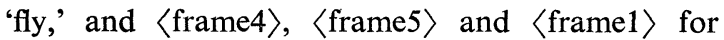
lexical information for the nouns, \{hikouki\} 'aero-

\begin{tabular}{|c|c|c|c|c|c|}
\hline \multicolumn{6}{|l|}{ unit11 } \\
\hline \multirow[t]{34}{*}{ event1 } & \multirow[t]{5}{*}{ frame2 } & MOOD & \multirow{5}{*}{\multicolumn{3}{|c|}{$\begin{array}{l}\text { DECLARAT } \\
\text { PAST } \\
\text { IPRF } \\
\text { NARRAT } \\
\text { NARRATOR }\end{array}$}} \\
\hline & & TENSE & & & \\
\hline & & ASPECT & & & \\
\hline & & STYLE & & & \\
\hline & & & & & \\
\hline & \multirow[t]{11}{*}{ frame3 } & TOK & \multirow{11}{*}{\multicolumn{2}{|c|}{\begin{tabular}{ll} 
tobas & \\
frame2 & \\
VST & \\
VC & \\
2 & 99 \\
frame1 & \\
LL1 & ningen \\
LL2 & buttai \\
02 & ningen \\
A & ningen \\
*0BJECT & \\
\multicolumn{2}{l}{ LOCATION }
\end{tabular}}} & \\
\hline & & MODAL & & & \\
\hline & & CATEG & & & \\
\hline & & & & & \\
\hline & & ACCENT & & & $\mathrm{H}$ \\
\hline & & *AGENT & & & \\
\hline & & PRED & & & basyo \\
\hline & & & & & basyo \\
\hline & & & & & buttai \\
\hline & & & & & frame4 \\
\hline & & & & & frame5 \\
\hline & \multirow[t]{6}{*}{ frame4 } & ток & \multirow{4}{*}{\multicolumn{3}{|c|}{$\begin{array}{l}\text { hikouki } \\
\text { ACCUSA } \\
\text { NST } \\
\text { hikouki }\end{array}$}} \\
\hline & & CASE & & & \\
\hline & & CATEG & & & \\
\hline & & & & & \\
\hline & & ACCENT & & 2 & $\mathrm{H}$ \\
\hline & & MODAL & frame2 & & \\
\hline & \multirow[t]{6}{*}{ frame5 } & TOK & nohara & & \\
\hline & & CASE & IN_LOC & & \\
\hline & & AKO & basyo & & \\
\hline & & ACCENT & & 1 & $\mathrm{H}$ \\
\hline & & CATEG & NST & & \\
\hline & & MODAL & frame2 & & \\
\hline & \multirow[t]{6}{*}{ framel } & & & & \\
\hline & & CASE & NOMINA & & \\
\hline & & & ningen & & \\
\hline & & ACCENT & & 1 & $\mathrm{H}$ \\
\hline & & CATEG & NST & & \\
\hline & & MODAL & frame2 & & \\
\hline
\end{tabular}

Fig. 2 Semantic frame representation of utterance:

/yuuziga noharade hikoukiwo tobasitexita/ 'Yuuzi was flying a model plane in the field.'

plane,' \{nohara\} 'field' and \{yuuzi\} 'Yuuzi' (a boy's name), respectively. From this expression the sentence generator ${ }^{14)}$ renders a skeltal phrase structure of a corresponding Japanese sentence. While oneto-one correspondence does not generally hold between the cases given by the lexicon and the surface case forms, we introduced the predicateme model, ${ }^{15}$ ) a version of valency grammar, to cope with it in generating case suffixes for noun phrases.

Thus, the syntactic structure in Fig. 3 shows an example of the final outcome thereof, corresponding to a sentence / yuuziga noharade hikoukiwo tobasitexita/ 'Yuuzi was flying an aeroplane in the field.' Each lexical morpheme is represented by a list composed of phonological form, a syllable number, an accent 


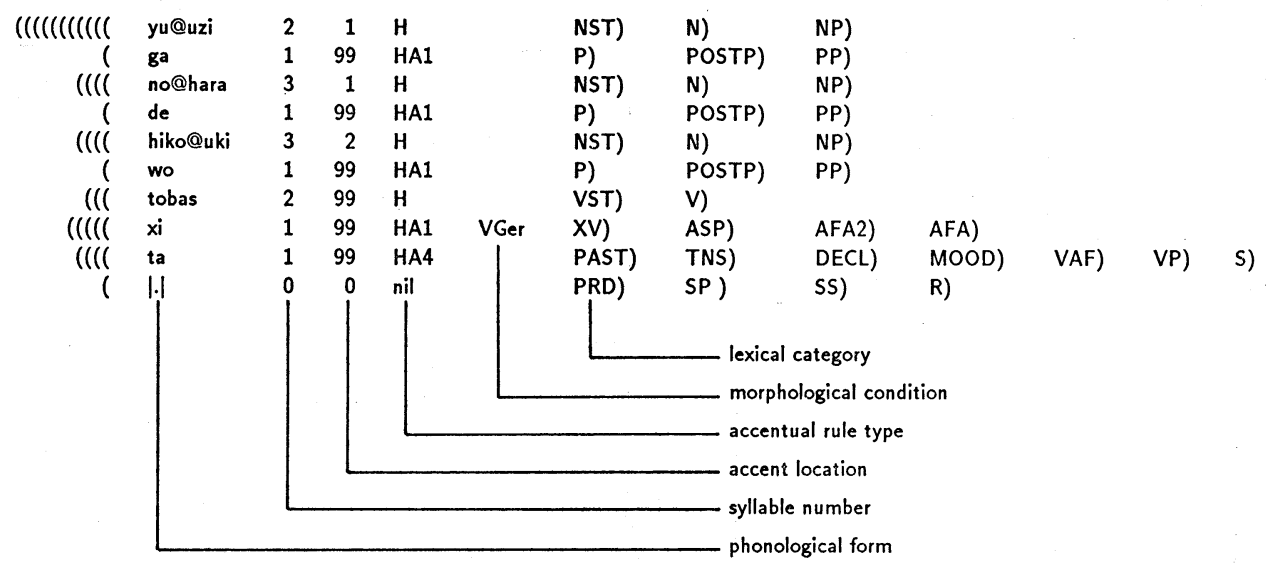

Fig. 3 Syntactic structure of sentence: /yuuziga noharade hikoukiwo tobasitexita/.

Table 1 Phonological rule classification.

\begin{tabular}{llll}
\hline Class & Subclass & Abbr. & \multicolumn{1}{c}{ Rule } \\
\hline Cyclic & Word formation & isa & Infinitival Suffix Affixation \\
& gsa & Gerundive Suffix Affixation \\
\cline { 2 - 4 } & Segmental & lvi & Low Vowel Insertion \\
& dva & Dental Voicing Assimilation \\
& vsm & Velar Stop Moraicization \\
& mcf & Moraic Consonant Formation \\
& fvi & Front Vowel Insertion \\
& nccent assignment & Nonmoraic Consonant Deletion \\
\hline & ta1 & Tail-dominant Accent Assignment 1 \\
& ta2 & Tail-dominant Accent Assignment 2 \\
& ta3 & Tail-dominant Accent Assignment 3 \\
& ha1 & Head-dominant Accent Assignment 1 \\
& ha2 & Head-dominant Accent Assignment 2 \\
& ha3 & Head-dominant Accent Assignment 3 \\
& ha4 & Head-dominant Accent Assignment 4 \\
\hline Noncyclic & hvd & High Vowel Devoicing \\
& Segmental & gsf & Glottal Stop Formation \\
& vsn & Velar Stop Nasalization \\
& dsa & Dental Stop Affrication \\
& dfp & Dental Fricative Palatalization \\
& gfp & Glottal Fricative Palatalization \\
& gfl & Glottal Fricative Labialization \\
& lvf & Long Vowel Formation \\
\hline & amd & Accent Movement from a Devoiced Syllable \\
& aml & Accent Movement in a Long Syllable \\
\hline & gsd & Glottal Stop Deletion \\
& dtg & Diphthongization \\
& bvd & High Back Vowel Deletion \\
\hline
\end{tabular}




\section{S. SATO: PHONOLOGICAL RULES: AN APPLICATION EXPERIMENT}

location, an accentual rule type, ${ }^{9)}$ morphological conditions $^{8)}$ and a lexical category, information all originated in the lexicon. To take an example of $\mid x i /$, an auxiliary for durative aspect, the list (xi 1 99 HA1 VGer XV) shows from left to right the following: the phonological form is $\mid x i /$ with $|x|$ representing a glottal stop. 1, 99 and HA1 are information utilized by the accentual rules, 1 denoting monosyllabicity, 99 unaccentedness, and HA1 the accentual rule it uses in stem-suffix concatenation, ${ }^{9}$ whereas VGer used in word formation rules shows that in stem-suffix concatenation, it requires a gerund of a verb stem in front of it, and XV indicates a lexical category, that is an auxiliary of verbal-inflectional type. ${ }^{8}$ With this structure fed to the phonological component, the three-tiered cyclic rules are activated between a pair of morphemes, each enclosed in a single pair of parentheses and adjacent to each other. We employ the following approximation for termination of cyclic application; the downstep is an accentual carryover observed in the prosody of a phonological phrase; a minor phrase is defined to be a phonological phrase containing a single downstep, ${ }^{16)}$ which can easily be found in utterance. Thus, we marked syntactic counterparts of minor phrases in input sentences, which roughly correspond to top-most postpositional phrases and lowermost verb phrases. As syntactic tree pruning proceeds, cyclic application stops when a marked syntactic category label is found. An outcome of cyclic rule application is now exposed to the noncyclic rules, which are also three-tiered as seen in Fig. 1. As opposed to the segmental nature of the lookup by the noncyclic segmental and phonetic rules over the sequence of phonetic symbols, contextual reference of the noncyclic accentual rules is syllable-based. However, in either way the noncyclic rules scan the sequence from left to right, looking for the right context through a-few-segment/ syllable wide window. Table 1 is the list of the phonological rules in the order of application as they operate in the system in Fig. 1. The three-letter abbreviations are for use in the programmed rules and traces of sample runs in the appendices.

The final output of the phonology is then accepted by the synthesizer providing it with corresponding spectral and temporal parameters supplied by the parameter file prepared in advance through acoustic analysis of Japanese. ${ }^{5,17}$ With a temporally concatenated parameter matrix the synthesizer ${ }^{18)}$ com- putes speech wave, yielding D/A converted Japanese speech; the segmental duration is computed by a statistical model proposed by Sagisaka and Tohkura, ${ }^{19)}$ whereas phrasal and sentential prosody is basically controlled by concatenation of linear functions. ${ }^{20 \text { ) }}$

\section{PHONETIC FROM GENERATION}

In order to evaluate the phonological rules and their application mechanisms in actual opeartion we performed a rule application experiment for phonetic form generation. This section presents application algorithms and data structures of the rules, and discusses the results.

\subsection{Algorithms and Data Structures}

Since the syntax produces tree-structured morphemes in their raw base forms, those making up a verb phrase have to go through three processes to render corresponding phonetic form: word formation (morphological verbal compounding), cyclic segmental and accentual rule application. In the particular case in Fig. 3 the morphemes in question are \{tobas $\}$ (verb stem 'to fly'), $\{\mathrm{xi}\}$ (durative auxiliary) and $\{\mathrm{ta}\}$ (past tense suffix) that should produce the phonetic form /tobasitexita/. These processes correspond to the three tiers of rules inside the loop in the flowchart in Fig. 1. Concatenation of these morphemes in the list structure in Fig. 3 takes place in this loop in the following manner: the innermost pair of parentheses enclosing a morpheme is deleted. For each morpheme embedded in multiple layers of parentheses this action is repeated until it is finally surrounded by a single pair. When it comes next to another morpheme also enclosed in a single pair, these two morphemes are concatenated with the pairs of parentheses deleted. As for the verb phrase in Fig. 3, the first concatenation occurs between $\{x \mathbf{x}\}$ and $\{t a\}$, deriving $\{x i t a\}$, then between $\{$ tobas $\}$ and $\{x i t a\}$, where the latter demands the former of its gerundive form. This demand meets the conditions of word formation rule $\langle\mathrm{gsa}\rangle$, and activates it, resulting in insertion of $\{t e\}$ (gerundive suffix). This is followed by application of segmental rule $\langle$ fvi $\rangle$ and accentual rule $\langle$ ha 4$\rangle$. The exact procedure performed by the rule application and morpheme concatenation engine is given in the lower half of the trace of a sample run of cyclic rule application in Appendix C. Similar processes are taken for the noun phrases in Fig. 3. 
Figure 4 shows this algorithm of rule application in Lisp function format. The function $\langle$ cyrac $\rangle$ is a function handling cyclic application, with its argument $\langle$ syn $\rangle$ being a sentence as in Fig. 3. For ease of understanding a sentence can approximately be a left-branching binary tree, where a node pointer goes from the root down into the leaves to look for terminal symbols. In the case where the pointer is at the node immediately dominating the terminal symbols, it has a list of all atoms of the form $(p n i r v c)$, where $p, n, i, r, v$ and $c$ denote the phonological form, syllable number, accent location, accentual rule, verbal compounding condition, and lexical category, respectively, and where $v$ can be null. The last $c$ is at the same time a node label underneath the present
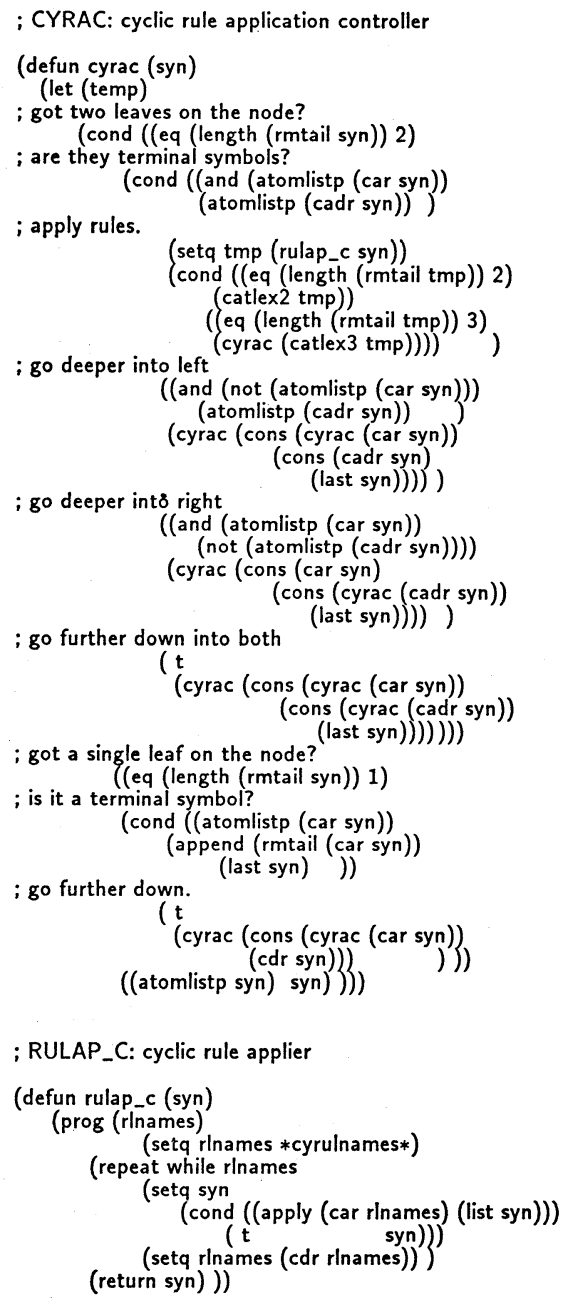

Fig. 4 Cyclic rule application algorithm. pointer location. Otherwise, wherever the pointer may be, it has either two or three elements in the list. (The last one denoting the node label, the node has one or two subordinate(s) underneath it.) A general form of the list being $\left(s_{1} s_{2} c\right), s_{1}$ and $s_{2}$ are lists, and $c$ is an atom denoting a lexical category, and $s_{2}$ can be null. There is an exceptional moment in the tree when a node dominates three subordinates; it is after a word formation rule has been applied and a gerundive/infinitival suffix is inserted in between, but that situation is resolved at the end of the cycle when a derived morpheme is made by concatenating the three. Thus, the subfunctions in 〈cyrac $\rangle$ have the roles as follows: 〈rmtail > removes the tail element $c$ of the list as the engine counts the number of the rest. 〈atomlistp〉 notifies whether the list is of

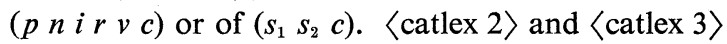
concatenate lexical morphemes at the end of the cycle. 〈rulap_c〉 is the cyclic rule applier that holds rule names in variable $\langle *$ cyrulnames* $\rangle$ and runs the rules at the pointed node in the order given in Table 1.

Figure 5 schematizes contraction of lexical information taking place in stem-suffix concatenation using a simple example of a noun stem /no@hara/ 'field' and a locative case suffix / de/ 'in.' While the segmental rules are not applied in the making of this particular noun phrase because of the mora structure granted in the lexical base, the accentual rules await this stem-suffix compound and start placing an accent on this derived stem using information carried in the lists of the original morphemes. In each of these particular lists the first element represents the phonological form with '@' indicating an accent on the vowel to its immediate left, and the second shows the number of syllables in the morpheme, whereas 1 and 99 in the third represent an accent on the first syllable and unaccentedness, respectively, and the fourth indicates the rule feature with $\mathrm{H}$ and $\mathrm{HA} 1$ indicating a stem and an accentual rule (ha1: headdominant accent assignment 1) to be applied in

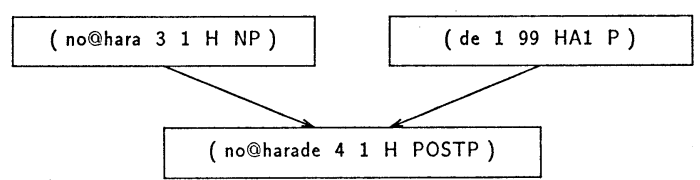

Fig. 5 Accentuation on noun phrase /noharade/ 'in the field.' 


\section{S. SATO: PHONOLOGICAL RULES: AN APPLICATION EXPERIMENT}

concatenation, respectively. Finally, the fifth gives a lexical or syntactic category. Thus, this /no@hara//de/ concatenation activates $\langle$ ha 1$\rangle$, yielding a derived stem at the lower half of Fig. 5. Exact procedure of this making is found in the upper half of the trace of a sample run of phonetic form generation in Appendix C.

Figure 6 (1) is an example of an output of cyclic rule application to a sentence, which now is to be exposed to the noncyclic rules. Figure 6 (2) is an intermediate expression of a list of segments and morpheme boundaries denoted by ' $=$,' to which the noncyclic segmental rules are applied looking from left to right for the context through a window of the length of two to four segments. The result of application of these rules is Fig. 6 (3). To name segmental changes there, they are $/ \mathrm{zi} /$ before $/ \mathrm{i} /$, palatalization by $\langle$ dfp $\rangle$; /gn/ non-phrase-initially, velar stop nasalization by $\langle v s n\rangle ; / h i /$ before $/ \mathrm{i} /$, palatalization by $\langle\mathrm{gfp}\rangle$; /iq/ between voiceless stops / $\mathrm{h} \mathrm{k} /$, devoicing by $\langle$ hvd $\rangle ; \mid x /$ before non-/a/, glottal fricative formation by $\langle$ gsf $\rangle ; / s i /$ before $/ \mathrm{i} /$, palatalization by $\langle\mathrm{dfp}\rangle$; and $/ \mathrm{iq} /$ between voiceless stops $/ \mathrm{s} t /$, devoicing by $\langle$ hvd $\rangle$. In the previous paper ${ }^{8)}$ we defined a set of distinctive features for the Japanese phonemes with these notations. Figure 6 (4) differs in the syllable based delimitation from Fig. 6 (3) after noncylic accent movement rule application although it is vacuous in this particular case. Figure 6 (5) gives the result of application of the phonetic rules. Consequently, the phonological component provides

\footnotetext{
(1) (yu@uziga no@harade hiko@ukiwo tobasitexita)

(2)(=yu@uziga=no@harade=hiko@ukiwo=tobasitexita=)

(3) (=yu@uzjigna=no@harade=hjiqko@okixo=tobasjiqtexita=)

(4) (=yu@u zji gna = no@ ha ra de = hjiq ko@okixo= to ba sjiq te xi ta =)

(5) (=y u@w zj i gna=no@ harade=hjiq $k \circ @ \circ k i \circ=t \circ b$ a sjiq t e y t a =)

(6) (yu@wzjigna no@harade hjiqko@okio tobasjiqteyta
}

Fig. 6 Segmental sequences in the noncyclic rule application processes. an input string for the synthesizer as in Fig. 6 (6). Exact noncyclic rule application procedures are found in the trace of a sample run for noncyclic rule application in Appendix D.

The rules are installed in the system in rewrite rule format, each represented by a three-element list. Figure 7 shows excerpts of the rules from each of the six layers. The general semantics of these rules is: the first element is replaced by the second in the asterisked position of the third provided that the context expressed in the third is satisfied. For the segmental rules any one of the three elements can be null; if it is the first one, insertion; the second, deletion; and the third, context-free operation. For the accentual rules only the third one can be null, in which case it is context-free. The distinctive features adopted in these rules are those defined elsewhere. ${ }^{8}$ In Fig. 7 word formation rule $\langle$ gsa_vb $\rangle$ (gerundive suffix insertion) inserts gerundive suffix $\{$ te $\}$ demanded by a verbal auxiliary like /i/ (durative auxiliary) in Fig. 3. Cyclic segmental rule $\langle d v a\rangle$ (dental voicing assimilation) replaces $/ t /$ with $/ d /$ as in $\{t a\}$ (past tense suffix) or $\{t e\}$ when preceded by a voiced consonant in the final segment of a consonant verb, as in /yom ta/ 'read-Past' becoming /yom da/. With an accent indicated by a '@,' cyclic accentual rule $\langle$ ha12〉 (head-dominant accent assignment 1) assigns an accent on noun phrase /no@harade/, which is made up of noun/no@hara/ with an accent on the first syllable and unaccented locative case suffix / de/ if the accent location of the noun $(i)$ is less than the number of syllables it has $(m)$. Noncyclic segmental rule 〈vsn〉 (velar stop nasalization) nasalizes $/ g /$ if it is not phrase-initial, with the segment immediately following [+seg] segment. Noncyclic accent movement rule 〈aml1〉 moves the accent placed on the subordinate part of a vowel sequence to the neucleus, as in /kai@ta/ 'wrote' changed to / $\mathrm{ka@ita/.} \mathrm{Noncyclic} \mathrm{phonetic} \mathrm{rule}$ $\langle$ dtg1〉 (diphthongization 1) diphthongizes a vowel

\begin{tabular}{|c|c|c|c|}
\hline gsa_vb: & ( nil & $(($ te 199 HA4 FV $))$ & $(((? ?$ ? ? V $)) *(($ ? ? ? ? VGer ? $))))$ \\
\hline dva: & $(((+a n+c r-c t))$ & $((\tan +\mathrm{cr}-\mathrm{ct}+\mathrm{vo}))$ & $(((-v c+c n+v o)) *))$ \\
\hline ha12: & $(((? \mathrm{~m} \mathrm{iH} ?)(? \mathrm{n}$ j HA1 ? )) & $((? m+n i \quad H ?))$ & $(($ i le $m)))$ \\
\hline vsn: & $(((+$ vo $-a n-c r))$ & $((+$ na -an $-c r))$ & $(((+s g)) *))$ \\
\hline aml1: & $(((C \vee \backslash @))$ & $((C \vee \vee @ I))$ & nil) \\
\hline dtg1: & $(((+h i-b a))$ & $((-v c+h i-b a))$ & $(((+v c)) *))$ \\
\hline
\end{tabular}

Fig. 7 Data structure of the phonological rules: excerpts. 
sequence, as in /ka@ita/ modified to /ka@ita/. Refer to Appendix A for the entire set of rules.

We have developed a rule compiler that translates these rules into Lisp functions so that the rule application engine can access them. Refer to Appendix $\mathrm{B}$ for illustrations of compiled form of the rules: cyclic segmental $\langle$ dva $\rangle$, accent assignment $\langle$ ha12〉 and noncyclic segmental $\langle v s n\rangle$, with their actual manipulation given in the above paragraph.

\subsection{Rule Application Experiment}

The rules in Table 1 are prepared as in Sec. 3.1 and installed in the system. A trace of a sample run of cyclic rule application is given in Appendix $\mathrm{C}$ for the sentence /yuuziga noharade hikoukiwo tobasitexita/ 'Yuuzi was flying a model plane in the field' whose syntax is displayed at the beginning of the trace as a returned value for variable $\langle$ syn11 $\rangle$. We see that the cyclic rule application engine composed of functions $\langle$ cyrac $\rangle,\langle$ rulap_c $\rangle$ and $\langle$ catlex $\rangle$ actually concatenates the stem-suffix compound when a cycle terminates. If not applied, a cyclic rule function returns 'nil,' which, however, is inhibited in the trace. As for the first three simplex noun phrases \{yuuzi ga\} 'yuuzi-Nominative,' nohara de\} 'field-Locative,' and \{hikouki wo\} 'plane-Accusative,' where mora structure is preserved from the lexical base, cyclic segmental rules do not apply, but accent assignment is made by 〈ha12〉, yielding/yu@uziga/, /no@harade/ and /hiko@ukiwo/. Note that the stem-suffix concatenation performed by 〈catlex〉 takes place for the first time on termination of the cycle although the syllable-related lexical values in suffixes are cleared after accentual rules are applied. After generating these noun phrases, $\langle\mathrm{gsa}\rangle$ is applied to insert $\{\mathrm{te}\}$ for gerundive compounding of \{tobas\} and \{xita\}, and $\langle$ fvi $\rangle$ further inserts $/ i /$ between $\langle$ tobas $\rangle$ and $\{$ te $\}$ to create moraicity. ${ }^{8)}\langle$ ha41 $\rangle$ is activated to produce unaccented subphrases /tobasite/ and /xita/ from \{tobas\} 'fly' and \{xita\} 'Durative-Past,' respectively, with the final outcome unaccented/tobasitexita/ using $\langle$ ha11 $\rangle$.

A trace of noncyclic rule application is shown in Appendix D, which is essentially the same as Fig. 6, but more detailed. It shows activation of eight noncyclic segmental and three phonetic rules: segmental rules: 〈hvd122 and hvd222〉 (high vowel devoicing) devoice two /i/'s in the contexts /h_k/ and /s_t $/$. $\langle$ dsf $\rangle$ (glottal stop formation) replaces $/ w /$ with $/ x /$. $\langle v s n\rangle$ nasalizes $/ g /$ and produces $/ g n / . \quad\langle\mathrm{dfp} 1\rangle$ and $\langle$ dfp2〉 (dental fricative palatalization) palatalize $/ \mathrm{s} z /$ in front of $/ \mathrm{i} /$. $\langle\mathrm{gfp}\rangle$ (glottal fricative palatalization) replaces $/ h /$ with $/ h i /$ before high front $/ i /$. $\langle$ lvf 1$\rangle$ (long vowel formation) changes /ou/ in /hiikouki/ into /oo/. Phonetic rules: 〈gsd (glottal stop deletion) deletes all $/ x /$ 's in the string. $\langle\operatorname{dtg} 1\rangle$ and $\langle\operatorname{dtg} 2\rangle$ (diphthongization) substitute $/ \mathrm{ei} /$ and /uv/ for /ey/ and /uw/, respectively. A noncyclic rule function returns the argument itself if not applied, which, however, is omitted in the trace in Appendix D.

\section{CONCLUSION}

In order to confirm validity of the phonological rules and their application algorithms, we installed the rules in the phonological component of a semantics-to-speech system implemented in the computer, and performed a phonetic form generation experiment using these rules. The rules are tiered based firstly on the segmental and accentual nature of data they handle, secondly on the cyclic rule application principle. Achievements we obtained are: (1) treestructured input sentences are converted to linearly aligned phonetic symbols in the simultaneous execution of the cyclic rule application and morphological stem-suffix concatenation. (2) With these tiers of rules and the cyclic rule application algorithm, integration of segmental and accentual phases of phonological processing is attained. (3) The rules are installed in the computer in a human-editable format, translated by a rule compiler into executable Lisp functions, tested and found to be valid.

\section{ACKNOWLEDGMENT}

The author wishes to thank Prof. Ken'iti Kido for his lasting support and encouragement throughout this work.

\section{REFERENCES}

1) D. H. Klatt, "Review of text-to-speech conversion for English," J. Acoust. Soc. Am. 82, 737-793 (1978).

2) H. Sato, Y. Sagisaka, K. Kogure, and S. Sagayama, "Investigation in Japanese text-to-speech conversion," Tech. Rep. Speech Acoust. Soc. Jpn. S82-08, 57-63 (1982) (in Japanese).

3) S. J. Young and F. Fallside, "Speech synthesis from concept: a method for speech output from information systems," J. Acoust. Soc. Am. 66, 685-695 (1979).

4) S. Yamamoto, N. Higuchi, and K. Matsuzaki, "Experimental system on speech synthesis from 


\section{S. SATO: PHONOLOGICAL RULES: AN APPLICATION EXPERIMENT}

concept," Proc. Autumn Meet. Acoust. Soc. Jpn. 2-3-16, 185-186 (1985) (in Japanese).

5) S. Sato and H. Kasuya, "Automatic translation/ speech synthesis of Japanese from written Esperanto incorporating a linguistic knowledge base editor," in European Conference on Speech Technology, Vol. 2, J. Laver and M. A. Jack, Eds. (CEP, Edinburgh, 1987), pp. 414-418.

6) C. L. Baker, Introduction to Generative-Transformational Syntax (Prentice-Hall, Englewood Cliffs, 1978), Chap. 7.

7) S. Sato, "Morphophonological derivation of Japanese predicate phrases: a phonological rule component for a semantics-to-speech system," J. Acoust. Soc. Am. 84, Suppl. 1, 25 (1988).

8) S. Sato, "Phonological rules for a semantics-tospeech system of Japanese: the segmental phase," J. Acoust. Soc. Jpn. (E) 11, 83-94 (1990).

9) S. Sato, "An accentual rule component for a speech synthesis system of Japanese," IEICE Tech. Rep. SP88-161, 45-50 (1989) (in Japanese).

10) R. Wilensky, LISP Craft (W. W. Norton, New York, 1984).

11) R. Nakagawa, Sorairono Tane (Fukuinkan, Tokyo, 1964) (in Japanese).

12) P. H. Winston and B. Horn, LISP, 2nd Ed. (Addison-Wesley, Reading, Mass., 1984), pp. 311320 .
13) T. Fujimoto, Lazura Semo (Libroteko-Tokio, Tokyo, 1977).

14) S. Sato and H. Komagata, "Application of the predicateme model to Japanese sentence generation," Proc. Autumn Meet. Acoust. Soc. Jpn. 3-4-7, 225226 (1986) (in Japanese).

15) S. Muraki, F. Aoyama, N. Rokujo, and K. Murata, "Description of case information in the dictionary," Trans. Comm. NLP Res., Inf. Proc. Soc. Jpn. 46-3, 1-8 (1984) (in Japanese).

16) E. Selkirk and K. Tateishi, "Constraints on minor phrase formation in Japanese," CLS 24 (Papers from 24th Annu. Reg. Meet. Chicago Linguist. Soc.), Part I: Gen. Sess., 316-336 (1988).

17) S. Sato, H. Kasuya, K. Murayama, and T. Sakamoto, "Speech synthesis system of Japanese from written Esperanto," IECE Tech. Rep. SP8610, 29-36 (1986) (in Japanese).

18) D. H. Klatt, "Software for cascade/parallel formant synthesizer," J. Acoust. Soc. Am. 67, 971-995 (1980).

19) Y. Sagisaka and Y. Tohkura, "Phoneme duration control for speech synthesis by rule," Trans. IECE Jpn. J67-A, 629-636 (1984) (in Japanese).

20) K. Hakoda and H. Sato, "Prosodic rules in connected speech synthesis," Trans. IECE Jpn. J63-D, 715-722 (1980) (in Japanese). 